\title{
Faraday Rotation Response to Coronal Mass Ejection Structure
}

\author{
E.A. Jensen • P.P. Hick • M.M. Bisi • B.V. Jackson • \\ J. Clover · T. Mulligan
}

Received: 14 December 2009 / Accepted: 13 March 2010 / Published online: 8 May 2010

(C) The Author(s) 2010. This article is published with open access at Springerlink.com

\begin{abstract}
We present the results from modeling the coronal mass ejection (CME) properties that have an effect on the Faraday rotation (FR) signatures that may be measured with an imaging radio antenna array such as the Murchison Widefield Array (MWA). These include the magnetic flux rope orientation, handedness, magnetic-field magnitude, velocity, radius, expansion rate, electron density, and the presence of a shock/sheath region. We find that simultaneous multiple radio source observations (FR imaging) can be used to uniquely determine the orientation of the magnetic field in a $\mathrm{CME}$, increase the advance warning time
\end{abstract}

Remote Sensing of the Inner Heliosphere

Guest Editors: M.M. Bisi, and A.R. Breen.

E.A. Jensen $(\varangle)$

ACS Consulting, 40 FM 1960 W PMB 370, Houston, TX 77090, USA

e-mail: eaj@acs-consulting.com

E.A. Jensen

MMT Observatory, Mt. Hopkins, Amado, AZ 85645, USA

P.P. Hick

University of California, San Diego, USA

e-mail: pphick@mail.ucsd.edu

M.M. Bisi

Institute of Mathematical and Physical Sciences, Aberystwyth, Ceredigion, SY23 3BZ, Wales, UK

e-mail: Mario.Bisi@aber.ac.uk

M.M. Bisi · B.V. Jackson · J. Clover

University of California/CASS, San Diego, USA

B.V. Jackson

e-mail: bvjackson@ucsd.edu

J. Clover

e-mail: jclover@ucsd.edu

T. Mulligan

Aerospace Corp, P.O. Box 92957, Los Angeles, CA 90009-2957, USA

e-mail: Tamitha.M.Skov@aero.org 
on the geoeffectiveness of a CME by an order of magnitude from the warning time possible from in-situ observations at $L_{1}$, and investigate the extent and structure of the shock/sheath region at the leading edge of fast CMEs. The magnetic field of the heliosphere is largely "invisible" with only a fraction of the interplanetary magnetic-field lines convecting past the Earth; remote sensing the heliospheric magnetic field through FR imaging from the MWA will advance solar physics investigations into CME evolution and dynamics.

Keywords Coronal mass ejections · Helicity, magnetic $\cdot$ Helicity, observations · Helicity, theory · Instrumental effects · Integrated Sun observations · Magnetic fields, corona · Magnetic fields, interplanetary $\cdot$ Magnetic fields, models $\cdot$ Polarization, radio $\cdot$ Radio scintillation $\cdot$ Rotation $\cdot$ Solar wind, disturbances $\cdot$ Solar wind, theory

\section{Introduction}

Coronal Mass Ejections (CMEs) consist of $10^{15}$ to $10^{16}$ grams of mass (Subramanian and Vourlidas, 2007) and "frozen-in" magnetic field erupting from the corona of the Sun achieving speeds between 450 and $2000 \mathrm{~km} \mathrm{~s}^{-1}$. At speeds exceeding the downstream solar wind speeds, a fast shock develops at the boundary between the CME and the downstream plasma. The Solar Energetic Particles (SEPs) accelerated by a shock can adversely affect spacecraft and initiate ionospheric disturbances (Kane, 2007), while the shock itself correlates with the onset of more intense geomagnetic storms (Badruddin and Singh, 2009). Intense geomagnetic storms generate significant radiation and cause the plasmasphere and ionosphere to expand. While spacecraft components can usually tolerate $100 \mathrm{krad}$ of radiation over their lifetime, a geomagnetic storm can generate $1 \mathrm{krad}$ thus reducing the lifetime of spacecraft; as a result, shielding is commonly implemented increasing the overall cost. For instance Telstar 401, an AT\&T television relay satellite, failed suddenly on 21 January 1997 following an Interplanetary CME (ICME) triggered geomagnetic storm in the middle of a Star Trek broadcast (Anselmo, 1997; Livesey, 1998). The expansion of the ionosphere increases drag on satellites and debris in Low Earth Orbit (LEO); this led to the early termination of the Solar Maximum Mission in 1990 (Smith, 1988), Skylab in 1979 (Glaser et al., 2003), and the U.S. Space Command had to recompute orbits for more than 1300 objects in 1989 (Odenwald, 1999). The ionospheric currents generated during the storms induce electrical currents in electricity lines: $2.6 \mathrm{kV}$ surges were recorded in the Atlantic cable between Scotland and Newfoundland in March 1940, and a geomagnetic storm caused a power blackout in Quebec on 13 March 1989. Ionospheric scintillations can disturb the amplitude and phase of traversing radio signals; this can cause navigation errors and signal loss (Nordwall, 1996). In 1998, satellite insurance companies paid $\$ 0.9$ billion in claims for satellite failures. By 2005 , $\$ 80$ billion worth of telecommunications services were carried by satellites. The Committee on the Societal and Economic Impacts of Severe Space Weather Events (2008) estimated a "severe geomagnetic storm scenario" could incur $\$ 1$ trillion in societal and economics damage.

\subsection{Geoeffectiveness Warning Time}

The geoeffectiveness of the interplanetary counterpart of CMEs, ICMEs (Lindsay et al., 1999), has been studied relative to their magnetic-field strength, orientation, speed, and the presence of a shock/sheath structure on its leading edge downstream in the solar wind. While the intensity of a geomagnetic storm is coincident with enhancements in magnetic-field 

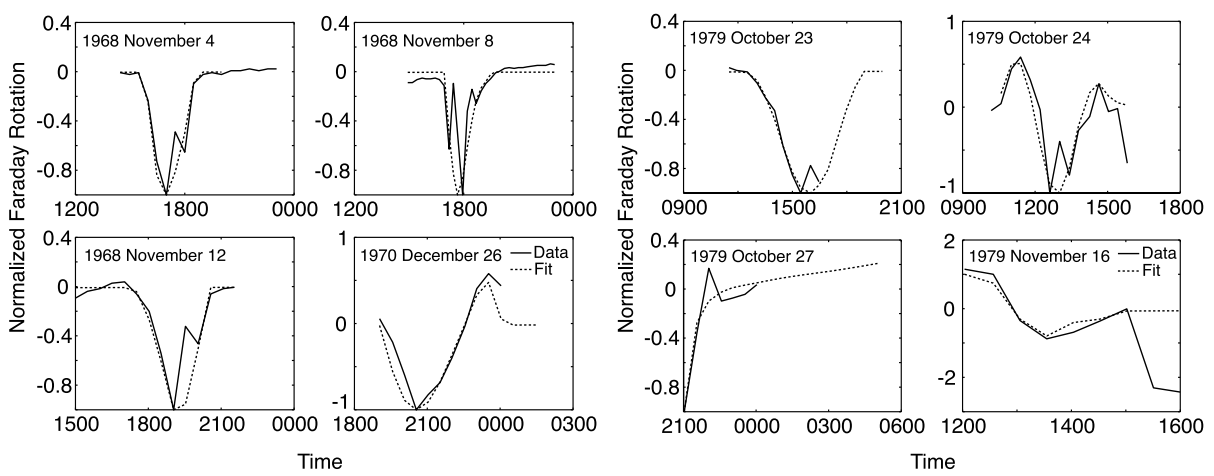

Figure 1 Normalized FR observations of magnetic-field configuration within CMEs. The signal offset distances (by date) are 11.5, 9.0, 6.5, 5.8, 7.0, 4.8, 5.8, and 3.4 solar radii. The FR observations have been normalized by 26.6, 44.0, 36.6, 10.2, 123.6, 42.2, 22.8, and 59.3 deg (adopted from Jensen and Russell, 2008).

strength of the ICME, the kinetic energy does not show a direct correlation (Badruddin and Singh, 2009). The magnetic-field orientation is significant to the geoeffectiveness of ICMEs; initial southward orientations (antiparallel to Earth's magnetic field) increase the probability of a geomagnetic disturbance. The presence of a shock/sheath region not only increases the amplitude of the geomagnetic disturbance, but it also could trigger its onset prior to the arrival of the magnetic flux rope. The ACE (Advanced Composition Explorer) and GOES (Geostationary Operational Environmental Satellites) satellites, upstream of the bow shock of the Earth's magnetosphere provide in situ data to NOAA's Space Environment Center with a 1 hour advance accurate warning on the geoeffectiveness of an incoming ICME with which to alert government and commercial interests (NOAA Space Weather Prediction Center, 2003); for fast ICMEs, this can be only 15 minutes. Increasing the warning time requires measuring the magnetic-field strength and orientation of the ICME and the existence of the leading shock/sheath much closer to the Sun.

\section{Faraday Rotation}

The only technique available to measure the magnetic field of the intervening heliosphere (of which few of the magnetic-field lines interact with the Earth) is by using the phenomenon of Faraday rotation (FR). Figure 1 shows a series of FR observations of CMEs collected with the Pioneer 6, Pioneer 9, and Helios spacecraft fit with a simple flux rope model (Jensen and Russell, 2008).

\subsection{Introduction to FR}

As an electromagnetic (EM) wave from a spacecraft, a bright pulsar, galaxy, or other natural radio source propagates through the birefringent plasma of the heliosphere, the plane of polarization of the EM wave rotates as a function of the magnetic field "frozen-in" to the plasma. Figure 2 illustrates the effect; when the magnetic field is directed along the k-axis, the rotation is in a right-handed sense increasing the angle as shown by the $\mathbf{E}$ field with time. The amount of FR is proportional to the integration of the component of the magnetic field along the line-of-sight (LOS) weighted by the electron density (Equation (1)). 
Figure 2 FR: the plane of polarization of an EM wave rotates in a right-handed sense (increasing angle) when the magnetic vector field of the medium is directed along the $k$-axis.

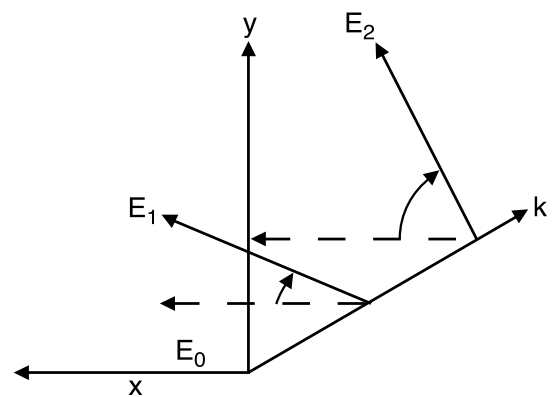

FR normalized to the wavelength of the traversing EM wave is represented by the Rotation Measure (RM):

$$
\begin{aligned}
& \left.\mathrm{FR}=\frac{A}{f^{2}} \int_{S C}^{\oplus} N \mathbf{B} \cdot \mathrm{d} \mathbf{s} \quad \text { [radians }\right], \\
& \mathrm{RM}=\mathrm{FR} / \lambda^{2} \quad\left[\text { radians } \mathrm{m}^{-2}\right], \\
& A=\frac{q^{3}}{8 \pi^{2} \epsilon_{o} m_{\mathrm{e}}^{2} c}=2.3648 \times 10^{4} \quad\left[\frac{\mathrm{m}^{2} \mathrm{rad}}{\mathrm{s}^{2} \mathrm{~T}}\right],
\end{aligned}
$$

where $f$ is the frequency of the signal in $\mathrm{Hz}, \mathrm{SC}$ is the spacecraft, $\oplus$ is the Earth, $N$ is the electron density in $\left[\mathrm{m}^{-3}\right], \mathrm{s}$ is the path of the signal in $[\mathrm{m}], q$ is the electron charge in $[\mathrm{C}]$, $m_{\mathrm{e}}$ is the mass of an electron in $[\mathrm{kg}]$, and $c$ is the speed of light in $\left[\mathrm{m} \mathrm{s}^{-1}\right]$.

\section{Modeling the FR Response from a CME/ICME}

When a CME/ICME, convecting radially outward from the Sun, crosses the LOS over a period of time, the FR changes due to changes in the electron density and the component of the magnetic field within the CME/ICME structure along the LOS; for the purposes of the case study in Section 4.1, the density of the CME/ICME is assumed to be the in-situ value measured at ACE (Skoug et al., 2004) with an $R^{-2}$ fall-off ( $R$ gives the heliocentric distance). The magnetic-field component of a CME/ICME consists of a magnetic flux rope discussed in Section 3.1. When bi-directional streaming electrons are observed along the magnetic-field lines traversing the Earth, it indicates that the field lines remain connected to the Sun. The dipole field line connecting the Sun and the axis of the "can" shown in Figure 3 illustrates this configuration of the magnetic flux rope; this figure will be discussed in more detail later.

\subsection{Ideal CME/ICME Magnetic Field}

Of all the CMEs ejected from the Sun, a fraction of them consist of magnetic fields strong enough to maintain the magnetic flux rope in a "force-free" configuration. Taylor (1986) demonstrated that the in-situ measurements of this configuration can be modeled with the outward magnetic pressure balanced by inward force from the curvature of the magneticfield lines with cylindrical symmetry (Equation (2)); this is called the Taylor state. The 
Bessel functions for this configuration are the axial $\left(J_{0}\right)$ and toroidal $\left(J_{1}\right)$ components of the field, which vary with distance from the center of the rope as shown in Figure 4.

$$
\begin{aligned}
& \mathbf{J} \times \mathbf{B}=0, \\
& \text { SO } \\
& \nabla \times \mathbf{B}=\mu_{o} \mathbf{J}=\alpha \mathbf{B}, \\
& \text { if } \\
& \alpha=\text { constant }, \\
& B_{z}=B_{0} J_{0}(\alpha r / R), \\
& B_{\phi}=B_{0} H J_{1}(\alpha r / R) \text {, } \\
& B_{r}=0 \text {, }
\end{aligned}
$$

where $J_{0}$ and $J_{1}$ are the Bessel function solutions, $\alpha$ is the constant determining twist of the flux rope, $H$ is the handedness of the rope ( +1 for right and -1 for left), $R$ is the radius of

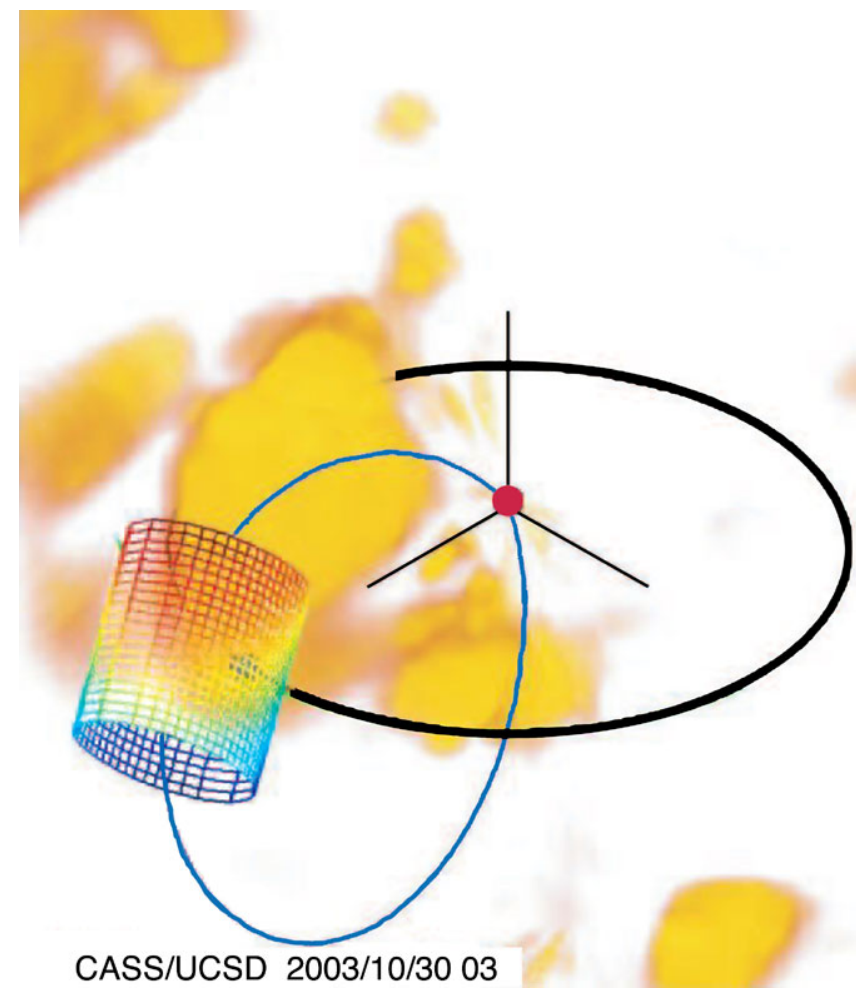

Figure 3 Magnetic-field configuration of the axial field to the flux rope of an ICME relative to the Sun. SMEI electron density reconstruction just as the ICME transits Earth. Densities are contoured between $10 \mathrm{~cm}^{-3}$ and $30 \mathrm{~cm}^{-3}$ with a $1 / R^{2}$ gradient removed (Jackson et al., 2006). "Can" diagram of the rope orientation for the magnetic flux rope fit is superposed; note the overall shape and orientation of the axial dipole field line is similar to that of the dense loop structure. 


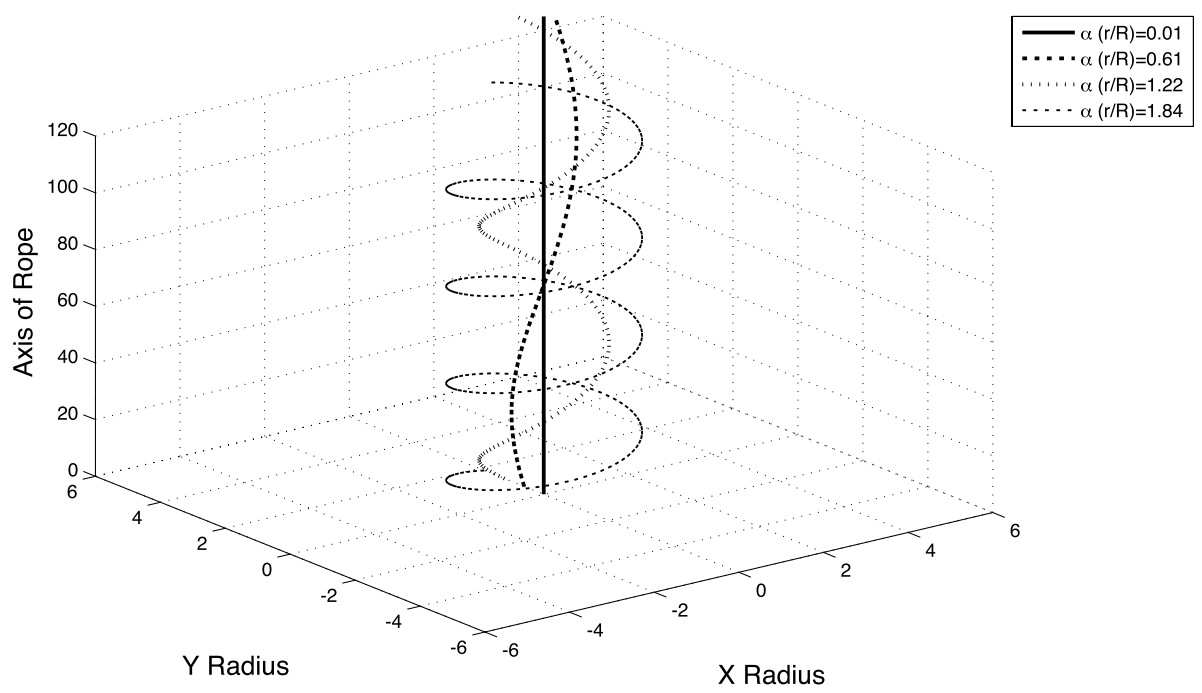

Figure 4 The magnetic-field line configuration of a Taylor state flux rope with Bessel function constant $\alpha=1.84$ where $r$ is the radius within the rope of radius $R$ (adopted from Russell and Mulligan, 2003).

the flux rope cylinder, and $\mathrm{r}$ is the radial position of a point in the flux rope. Note that $\alpha$ was set to 2.0 for all the fits except 24 October 1979 (where it was set past the pinch point where the axial field in the configuration reverses). The magnetic-field configuration at a particular radius within the flux rope $(r)$ is given by $\alpha(r / R)$. For instance, as shown in Figure 4 the field lines near the center have very little twist; in contrast at the edge of the flux rope, $r=R$, the field line is strongly twisted.

\subsection{CME/ICME Flux Rope Coordinate System}

We generate the full range of possible FR signatures for a CME/ICME crossing the LOS using the following coordinate system. As shown in Figure 5, the $x$-axis extends along the LOS from the source toward Earth. The $y$-axis is perpendicular to the LOS from the center of the Sun to the point of closest approach; this is the direction of the solar wind. The $z$-axis completes the right-handed coordinate system. Thus, for a LOS in or near the ecliptic, the $z$-axis is normal to the ecliptic plane pointing north above the west limb of the Sun and south above the east limb. The orientation of the axis of the flux rope within this coordinate system is defined with spherical angles used in the cone-clock system: the clock angle increases from 0 degrees along the $y$-axis to 90 degrees along the $z$-axis, and the cone angle is the offset angle from the $y-z$ plane. The cone angle is 0 degrees along the $x$-axis and 90 degrees in the $y-z$ plane. Table 1 gives the values for the fits shown in Figure 1 using this coordinate system. Figures 6 and 7 illustrate the range of FR signatures that result from different flux rope orientations. The cone angle is given by $\theta$, and the clock angle is given by $\phi$. Assuming the flux rope in Figure 5 is right-handed (RH), the FR signature produced is shown by $\phi=90$ and $\theta=90$ in Figure 6 . Note that initialization of the fit routine for Figure 1 (described in the following section) required the initial orientation angle to be selected within the correct quadrant using these templates. 
Figure 5 The geometry of a $\mathrm{CME}$ crossing at initiation. The position of the spacecraft $\left(R_{\mathrm{SC}}\right)$, the signal path (SP), the CME track (its position when it crosses the LOS) $(C)$, the perpendicular to the axis of the CME and the signal path $\left(X_{\text {ing }}\right)$, the vector to the point of closest approach (IP), and the angle from IP to $C$ (CA) are shown. The cone angle and clock angles of the CME shown are $\theta=\phi=90$ degrees as discussed in Section 3.2 (adopted from Jensen and Russell, 2008).

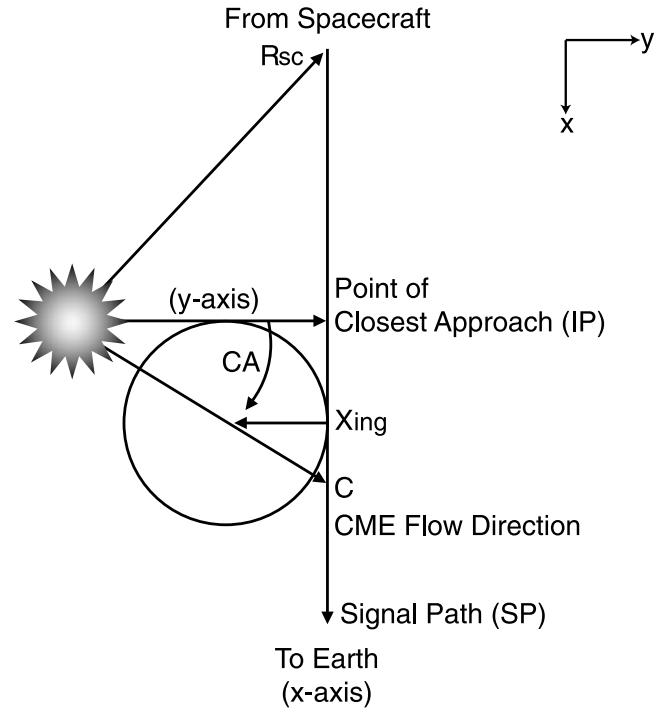

Table 1 The orientations of the flux rope fits to the observed FR transients assuming RH ropes (Jensen and Russell, 2008).

\begin{tabular}{llccc}
\hline Date & limb & $\theta(\mathrm{deg})$ & $\phi(\mathrm{deg})$ & CA (deg) \\
\hline 4 November 1968 & west & 179.5 & 168.6 & \pm 32.3 \\
8 November 1968 & west & 146.4 & 205.9 & \pm 61.8 \\
12 November 1968 & west & 151.1 & 171.0 & \pm 8.1 \\
6 December 1970 & east & 104.7 & 82.2 & \pm 29.0 \\
23 October 1979 & west & 152.2 & 160.2 & \pm 35.1 \\
24 October 1979 & west & 151.4 & 151.4 & \pm 28.6 \\
27 October 1979 & east & 81.1 & 83.5 & \pm 88.7 \\
16 November 1979 & west & 55.8 & 266.1 & \pm 50.6 \\
\hline
\end{tabular}

\subsection{CME/ICME Crossing FR Model}

Using the Taylor state flux rope model for the magnetic-field configuration in a CME/ICME, the FR measurements of CMEs crossing the LOS were investigated to determine if the orientations of the magnetic fields could be uniquely determined (Jensen and Russell, 2008). The FR model consisted of fitting the parameters shown in Figure 5. The flux rope flows outward in the radial direction designated by $\mathrm{C}$ at an angle, CA, from the Sun-to-point-ofclosest-approach vector. Note that the CA angle is ambiguous; a single LOS FR measurement, without information from other instruments observing the corona, cannot distinguish whether the crossing structure is moving towards or away from the Earth. As shown in the figure, the axis of the flux rope is oriented perpendicular to the Sun-LOS plane, and the parameter $\left(X_{\text {ing }}\right)$ designates the minimum distance between the LOS and the axis of the flux rope. When this distance is equal to the radius of the flux rope, the crossing of the LOS begins. Further discussion of the fitting process can be found in Jensen and Russell (2008). Table 2 gives the measured columnar electron densities, the impact parameter (offset distance) of the closest approach of the LOS, and the apparent velocity of the CMEs 

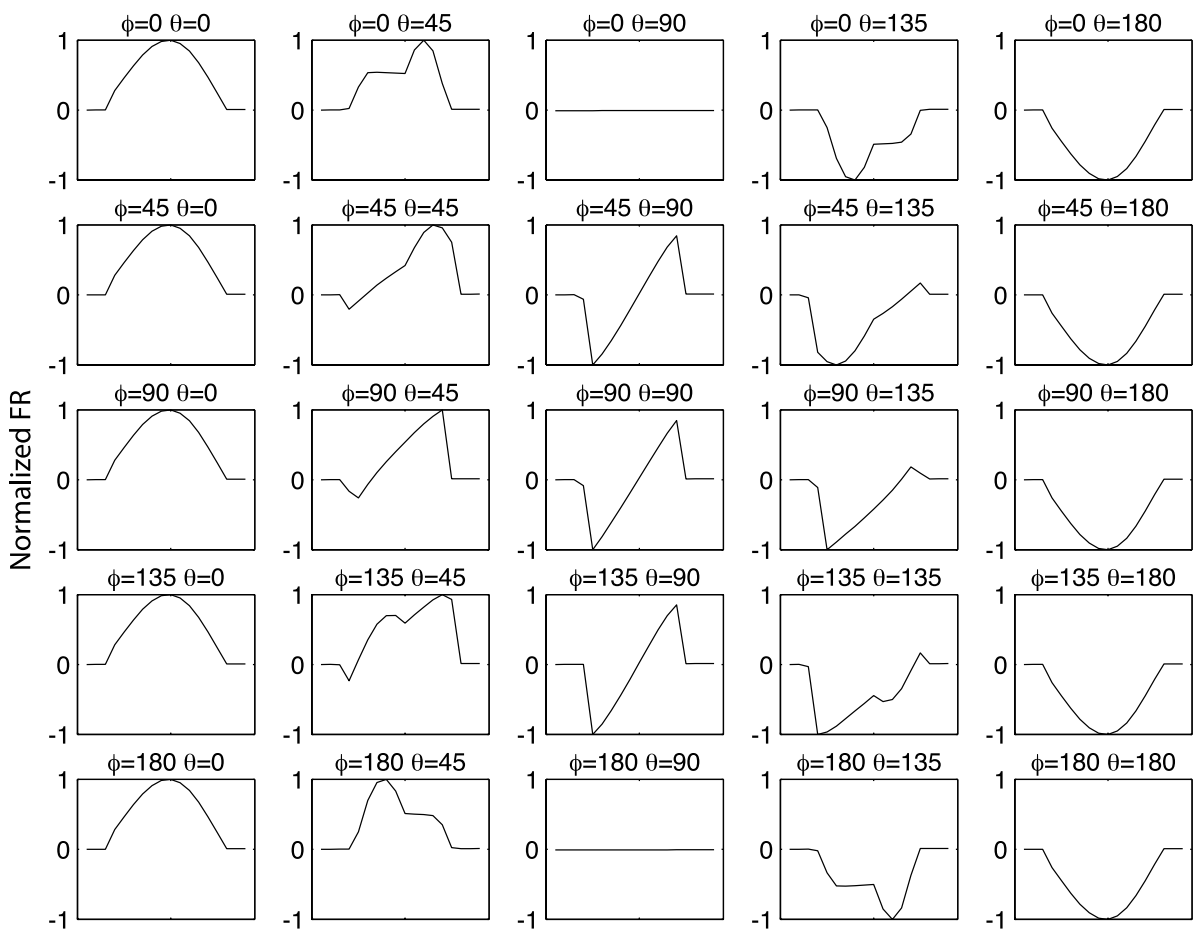

Time

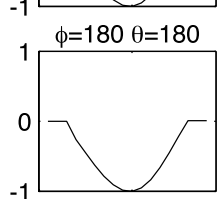

Figure 6 Range of normalized FR configurations from RH flux rope crossings with time through clock angles $(\phi)$ from 0 to 180 degrees. Note that Rotation Measure (RM) values for the observations shown in Figure 1 can range from 600 to $7300 \mathrm{deg} \mathrm{m}^{-2}$.

Table 2 The rope radii $(R)$ of the fits versus the columnar electron densities $(I)$, impact parameters (IP), and observed velocities $\left(\mathrm{Vel}_{A}\right)$ (Bird et al., 1985; Stelzried, 1968; Levy et al., 1969; Cannon, 1976) from Jensen and Russell (2008).

\begin{tabular}{llrll}
\hline Date & $I 10^{20} \mathrm{~m}^{-2}$ & IP Rs & $\mathrm{Vel}_{A} \mathrm{~km} \mathrm{~s}^{-1}$ & $R \mathrm{Rs}$ \\
\hline 4 November 1968 & & 11.5 & $930(450,1170)$ & $4.7(2.3,6.0)$ \\
8 November 1968 & & 9.0 & 1400 & 4.7 \\
12 November 1968 & & 6.5 & $640(440,820)$ & $2.3(1.6,3.0)$ \\
6 December 1970 & & 5.8 & 120 & 1.5 \\
23 October 1979 & $4.4(4.1,4.4)$ & 7.0 & $150(60,220)$ & $1.0(0.17,1.6)$ \\
24 October 1979 & $2.7(2.2,3.7)$ & 4.8 & $160(80,210)$ & $1.8(0.73,2.4)$ \\
27 October 1979 & & 5.8 & $260(60,370)$ & $7.5(4.9,15)$ \\
16 November 1979 & & 3.4 & 1000 & \\
\hline
\end{tabular}

from observations (Bird et al., 1985; Stelzried, 1968; Levy et al., 1969; Cannon, 1976). The radius of the flux rope from the model fit is presented. 

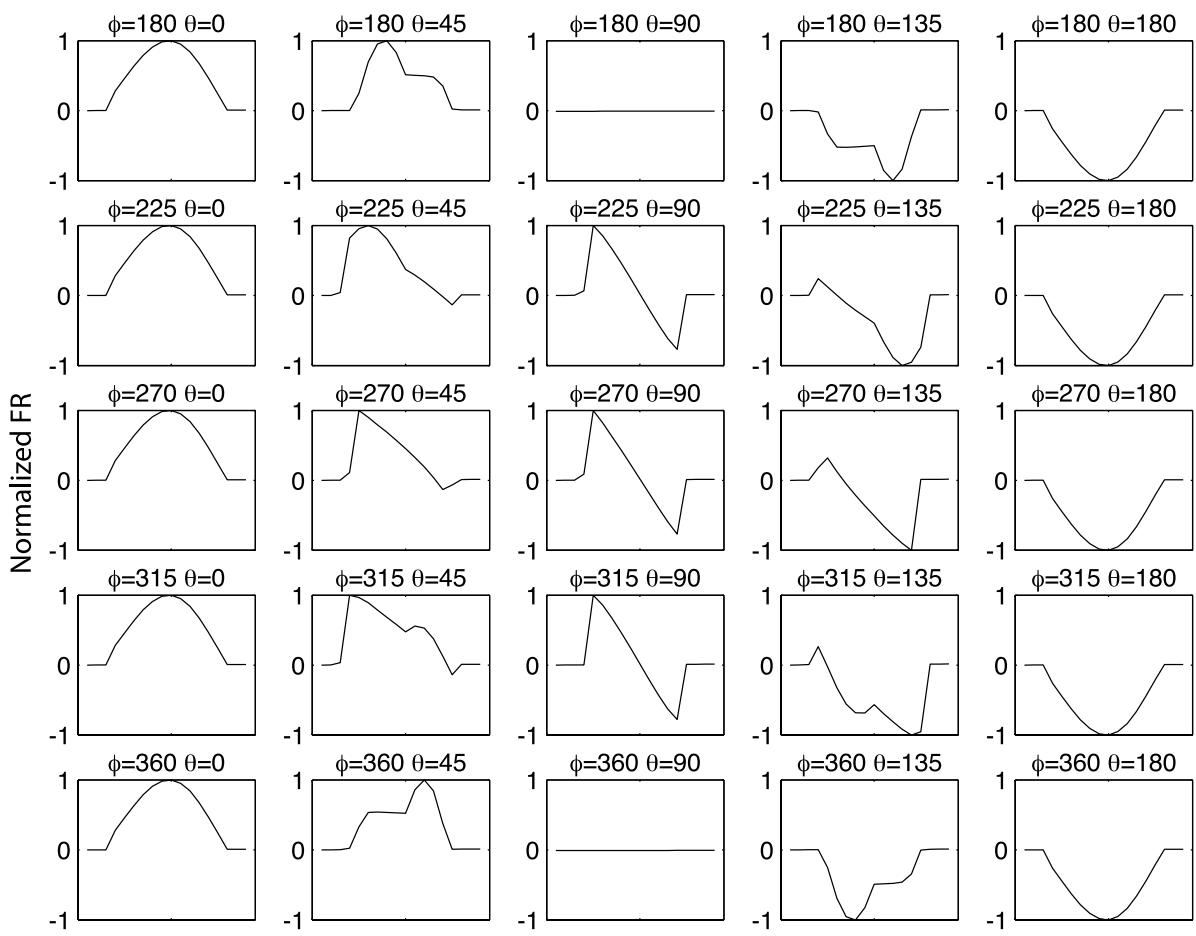

Time

Figure 7 Range of normalized FR configurations from RH flux rope crossings with time through clock angles $(\phi)$ from 180 to 360 degrees. Note that RM values for the observations shown in Figure 1 can range from 600 to $7300 \mathrm{rad} \mathrm{m}^{-2}$.

\subsection{FR Images}

We note that the configurations shown in Figures 6 and 7 are for RH flux ropes; left-handed (LH) flux ropes are characterized by the same signatures reversed along the abscissa. However, this introduces a level of ambiguity to these fits as noted by Jensen and Russell (2008). For instance, the rope shown in Figure 5 produces the same signature from the toroidal component of the magnetic field (a sigmoid with an initial decrease) as a LH rope with a clock angle of 270 degrees and the same cone angle. A second, simultaneous observation along a separate LOS which measures the unambiguous direction of the axis of the flux rope eliminates this ambiguity between flux rope handedness and orientation. The Murchison Widefield Array (MWA), presently under construction and beginning testing operations, will measure the FR from multiple natural sources simultaneously creating an image which can eliminate the ambiguity. Figure 8 illustrates the potential in RM imaging of the plane-ofsky (POS) of the 1979 October 27 Helios CME. The background RM field is removed, the electron density is $5 \mathrm{~cm}^{-3}$ that falls off as $R^{-2}$ with heliocentric distance, the magnetic-field structure is the Bessel function cylinder described above, the height of the cylinder is twice the radius, the center of the cylinder is at $0.7 \mathrm{AU}$, and its magnetic-field strength is $100 \mathrm{nT}$. The orientation of the flux rope is clear in this idealized case. While the Helios observation on the east (left) equator only observed a negative FR that grew weaker as the structure expanded and convected outward, it was not clear from the observation whether it was a 

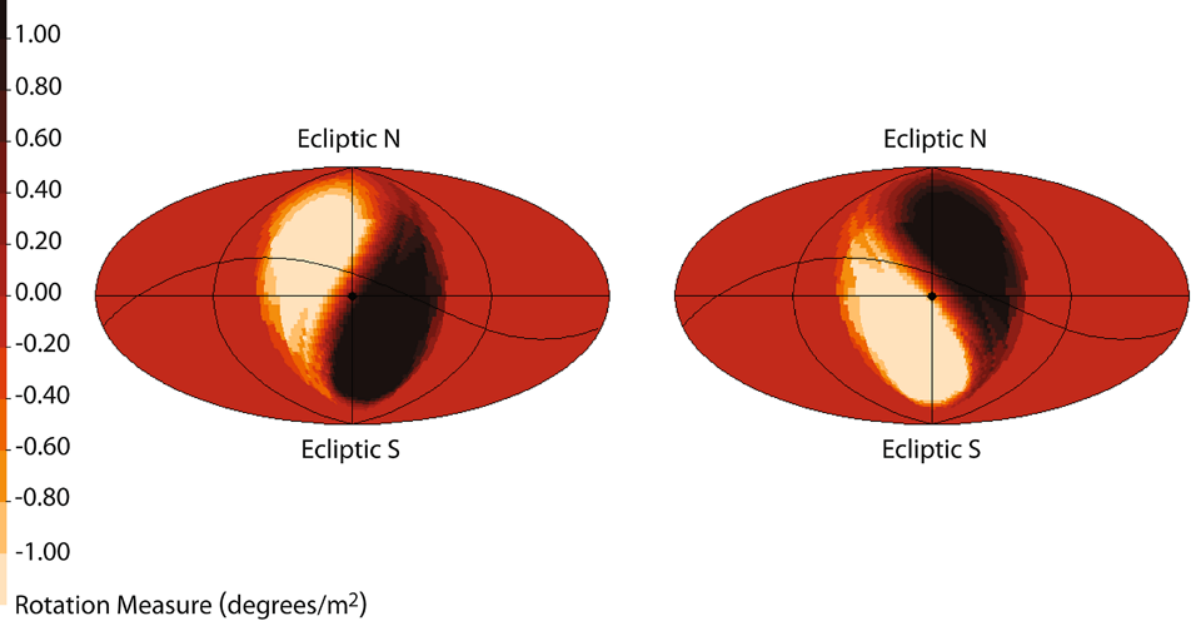

Figure 8 A Hammer-Aitoff simulation of a MWA RM image of the Helios 27 October 1979 CME. The center of the CME is located at 0.7 AU; the left- (right-) hand side shows the image of a left- (right-) handed flux rope capable of reproducing the observation. Note that Helios was located six solar radii off of the east (left) limb equator of the Sun. The image uses 10 kpixels to simulate the density of sources expected by the MWA and shows the plane-of-sky (POS) with the inner line at 90 degrees elongation encompassing the dayside hemisphere. The region between this line and the outer ellipse is the nightside hemisphere. The Sun is at the center, the horizontal line is the ecliptic, and the offset curved line is the celestial equator.

southward ecliptic oriented (parallel to the $z$-axis of the model coordinate system) RH flux rope (Figure 8, right) or a northward ecliptic oriented (270 degree clock angle in the model coordinate system) LH flux rope (Figure 8, left). The northern-most and southern-most portions of the structure are dominated by the axial component of the flux rope. A northward oriented flux rope's axial magnetic field is antiparallel to the LOS from a source in that position in the POS, thus generating a negative FR and visa versa.

Note that this structure is a cylinder, while actual flux ropes have a curved axis; a more accurate representation of the flux rope would show the axis of the rope extending a little further to the north and the south and would fractionally reduce the spatial extent of the toroidal-dominated RM regions. Clearly a MWA-type FR image can eliminate the inherent ambiguity between magnetic field orientation and handedness within CME/ICMEs. Cautionary note: the magnitudes for the amount of RM measured in the simulation are only accurate at the offset at which the FR measurements were made; in the case of the 27 October $1979 \mathrm{CME}$, its $\mathrm{RM}$ was $1340\left[\mathrm{deg} \mathrm{m}^{-2}\right.$ ] at an offset of 5.8 solar radii. The solar wind expansion applied to the structure for simplicity has been noted by various authors to be oversimplified (i.e. Mulligan, 2002; Forsyth et al., 2006). Actual FR images are needed to make accurate measurements of the CME expansion.

\subsection{MWA}

Located in a quiet radio frequency region of Australia, the MWA is capable of observing natural radio sources across a range of frequencies from 80 to $300 \mathrm{MHz}$ (in contrast, spacecraft radio frequencies are either 2.3 or $8 \mathrm{GHz}$ ). The physical layout of the array allows observing all the sources in $1000 \mathrm{deg}^{2}$ of the POS simultaneously at a particular predetermined observing frequency. The available sources for observing are determined by the rotation and 
Figure 9 ACE magnetic-field data of the 2003 Halloween event (Skoug et al., 2004) fit using the Mulligan - Russell model (Mulligan and Russell, 2001) for magnetic-field orientation at 1 AU. The GSE clock and cone angles for this fit are -108 and 129 degrees, respectively, indicating the rope axis is inclined to the ecliptic plane with a magnetic-field strength of $60 \mathrm{nT}$, and closest approach of the magnetic rope axis is $25 \%$ of the rope radius to the dusk side of the Earth. The current densities for this fit also indicate a nearly force-free structure. The circles are the Taylor state flux rope model magnetic fields for the ICME in GSE coordinates from the simulations.

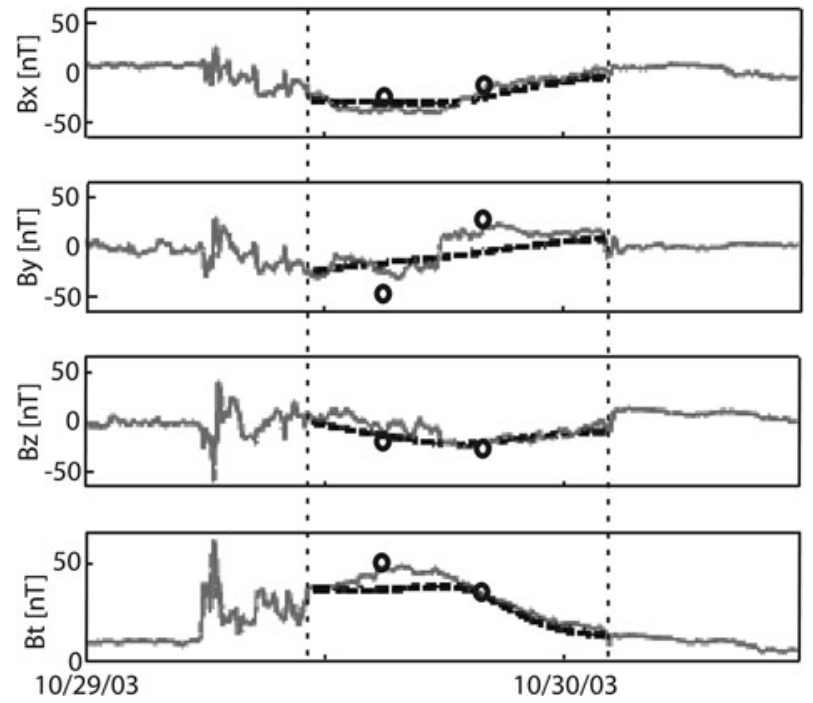

position of the Earth throughout the year. Salah et al. (2005), estimates that one source per 4 $\mathrm{deg}^{2}$ can be observed from a survey in the $340-370 \mathrm{MHz}$ frequency range of sources with "readily measurable Faraday rotation"; the degree of polarization of sources decreases with decreasing frequency (Spangler, 2006, personal communication).

Imaging the contribution to FR images from CMEs with the MWA will require proper calibration and removal of the background heliosphere and ionosphere; these contributions at large offsets from the Sun (i.e. 0.4 AU) can exceed the expected FR contribution from the CMEs themselves. As the MWA begins operations and develops system-specific calibration techniques, we recommend its investigations into CME propagation in the heliosphere initiate at the $300 \mathrm{MHz}$ range thus maximizing the CME contribution to the signal at the smallest possible solar offset (between 15 and 30 solar radii depending on available computer processing resources).

\section{ICME Case Study: 2003 Halloween Events}

As discussed previously, the existence of a shock at the boundary between the ICME and the downstream plasma increases the probability that the arriving ICME will be geoeffective. To investigate the advance warning application of MWA-type FR images, we performed a case study on the Halloween event from 2003.

\subsection{Halloween Inversions}

At 11:02 UT on 28 October 2003 a Type II radio burst reached Earth from the third-most powerful solar X-ray flare ever recorded (category 4B/X17.2). The CME originating during this flare erupted from AR 10486 located at S16 E08. The interplanetary (IP) shock driven by the ICME had a transit time to Earth of 20 hours (Cliver and Svalgaard, 2004). The ACE spacecraft, located at the $L_{1}$ libration point sunward of the Earth measured the IP shock and ICME driver as it passed the Earth on 29 October 2003. Figure 9 shows the ACE magneticfield data in GSE (Geocentric Solar Ecliptic) coordinates during the ICME passage. The 
leading and trailing edges of the flux rope cylinder are marked by vertical dashed lines. The Solar Mass Ejection Imager (SMEI) successfully recorded all-sky images during this time, except for a one-day outage on 30 October 2003 just after the bulk of the ICME event arrived at Earth (Jackson et al., 2006).

\subsection{Mulligan - Russell Model Inversion}

A non-force-free flux rope model used to obtain the geometry and properties of flux rope ICMEs was inverted with the ACE magnetic-field data (black dashed line in Figure 9). The model has an axial magnetic-field component that falls off with an exponential dependence from the axis of the rope. The toroidal (or poloidal) field increases as one minus an exponential dependence so that it maximizes at the rope edge. The model flux rope is fit to the data using a downhill simplex inversion technique (Nelder and Mead, 1965) that varies the fitting parameters in an orderly manner. Further details of the model can be found in Mulligan and Russell (2001). The GSE latitude and longitude angles for this fit are -42 and 201 degrees indicating the rope axis is inclined to the ecliptic plane with a magnetic-field strength of $60 \mathrm{nT}$, and closest approach of the magnetic rope axis shown in Figure 3 is $25 \%$ of the rope radius to the dusk side of the Earth. Note that GSE longitude is the angle from the $x$-axis to the $y$-axis, and the latitude is the angle from the $x-y$ plane.

\subsection{SMEI Tomographic Inversion}

Figure 3 shows the three-dimensional (3-D) electron density field reconstructed from SMEI data just as the ICME transits Earth (Jackson et al., 2006). Densities between $10 \mathrm{~cm}^{-3}$ and $30 \mathrm{~cm}^{-3}$ with a $1 / R^{2}$ gradient removed are shown. Overlaid on the SMEI density is the "can" diagram for the model inversion. The 3-D geometry and orientation of the flux rope "can" is shown at the same time and from the same point of view as the SMEI reconstruction to enable direct comparison. The dipole field line threading the "can" is constrained to be tangent to the magnetic rope axis in the ecliptic plane. The location of the Earth and Sun are indicated, and Earth's orbit is shown by the ellipse. Notice the dominant 'loop' structure and the dipole field line are clearly seen passing northeast of the Earth. The dipole field line direction is similar to the density enhancement 'loop' in the 3-D reconstruction from SMEI and is consistent with the Interplanetary Scintillation (IPS)-SMEI comparison study (Tokumaru et al., 2007).

\section{RM Image Simulation Construction}

Using the orientation from the Mulligan-Russell non-force-free flux rope fit to the magnetic-field component of the Halloween ICME, we introduce a Taylor state force-free magnetic-field configuration in our simulation for generating an ideal MWA FR measurement. Figure 9 illustrates the difference in the magnetic-field configuration from the in-situ data with this model. The GSE $B_{x}, B_{z}$, and $B_{t}$ align closely with the Mulligan-Russell model fits. The GSE $B_{y}$ magnetic-field strength is significantly stronger than the fit. However, the FR measurement is dominated by the component of the field parallel to the GSE $B_{x}$ direction; therefore, this difference in $B_{y}$ is not considered a problem for our present application. 
Figure 10 Top: Illustration of the "shock/sheath" shell. The shell extends for the length of the flux rope out of the plane; it is one CME/ICME radius thick, and smoothly extends out to two $\mathrm{CME} / \mathrm{ICME}$ radii on the sides. Density $(N)$ in the shell decreases with distance from the CME/ICME. Bottom: The RM for the simulated shock/sheath shell of the 2003 Halloween event; the center of the ICME (not visible) is at $0.4 \mathrm{AU}$. Note that values in excess of the color scale are either black (positive) or white (negative).
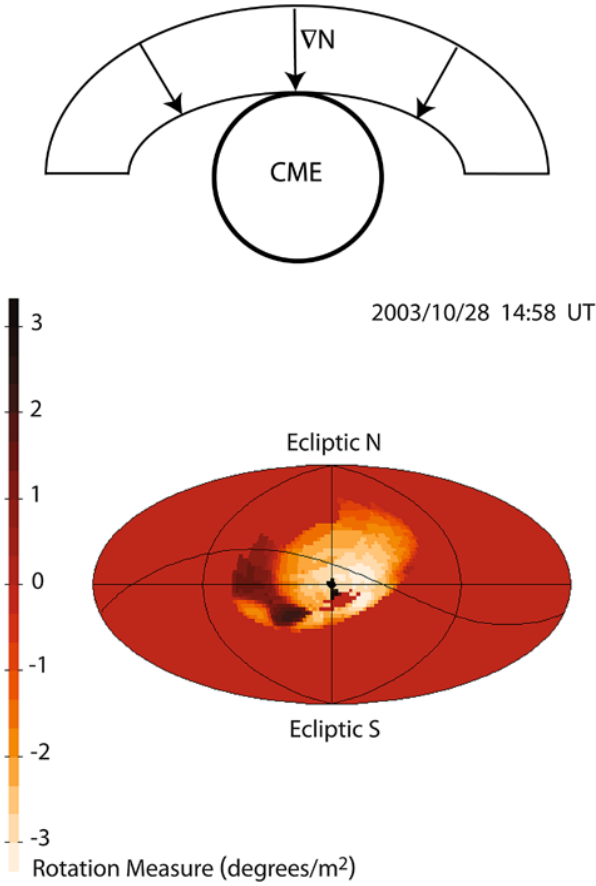

Cautionary note: the magnitudes for the amount of RM measured in the simulation are only accurate at $1 \mathrm{AU}$. The solar wind expansion applied to the structure for simplicity has been noted by various authors to be oversimplified (i.e. Mulligan, 2002; Forsyth et al., 2006). Actual FR images are needed to make accurate measurements of the CME expansion. Also note that the in-situ proton density measured at ACE was approximately $5 \mathrm{~cm}^{-3}$, while the electron density measured at Geotail was $2-5$ times greater indicating that the RM within the CME may be greater (Skoug et al., 2004). Because electron density strongly influences the measured amount of FR, we assume that the bottom image in Figure 11 is a lower boundary.

\subsection{Shock/Sheath Construction}

As shown in Figure 9, the $2000 \mathrm{~km} \mathrm{~s}^{-1}$ ICME developed a shock and sheath downstream on the leading edge. Shock/sheath structures consist of enhanced magnetic field and electron density (not necessarily in the same region), thus making a significant contribution to the measured FR of the region. Studies into shock/sheath structures leading ICMEs indicate that the magnetic-field configuration and electron density distribution in the region are strongly dependent on the spatial structure of the ICME in the heliosphere with the deflection flows passing from the faster areas of the structure to slower (Liu et al., 2008) as well as away from the structure.

To create an obstacle downstream of the CME/ICME to study the Faraday rotation response to a shock/sheath-like structure, we created circular shell aligned with the axis of the CME/ICME (Figure 10, top). The sheath region from in-situ observations is thin leading the ICME and extends outward beyond the ICME by the deflected plasma flow. We simulated this by setting the thickness of the shell to the radius of the ICME in the portion leading 
Figure 11 Simulated MWA RM images of the Halloween event ICME on 29 October 2003.
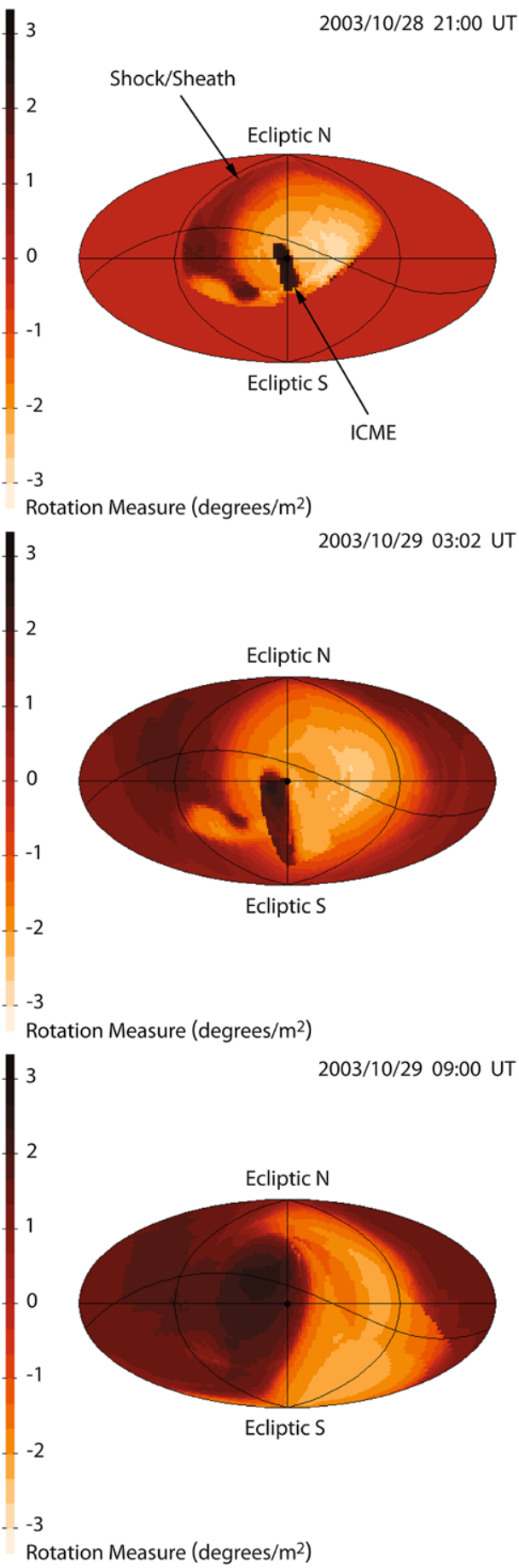
the ICME and extended the shell outward from the ICME to two ICME radii on all sides. Because observations of the shock/sheath magnetic-field structure in the heliosphere other than along the Sun-Earth line are unavailable, the magnetic-field values for the region of space occupied by the shell were calculated from the Stanford Current-Sheet Source-Surface (CSSS) model propagated outward with the solar wind (Dunn et al., 2005). Within the shell, the density is greatest next to the ICME and falls off with distance towards the shock; we increased the density by a factor of two next to the ICME and allowed it to fall off with an inverse square to the ideal density of the surrounding undisturbed solar wind plasma. Figure 10 (bottom) shows the structure of the simulated "shock/sheath region" shell for the Halloween event. The center of the ICME behind the sheath is at $0.4 \mathrm{AU}$; at this offset distance, the RM contribution from the flux rope is significantly less than the "shock/sheath" shell and not visible.

\subsection{Time Sequence RM Images}

The magnetic flux rope configuration with the "shock/sheath region" shell for this event is shown with time in Figure 11 as a sequence of RM images that could be obtained from the MWA at a 50 minute cadence; note that the MWA cannot observe FR through upstream solar wind for periods longer than 6 hours a day and that values in excess of the color scale are either black (positive) or white (negative). Although the plasma of the shell dominates the negative FR response, the positive FR component of the flux rope dominates the signal above the east limb of the Sun. This positive component, both along the axial and toroidal fields of the flux rope, allows the unique measurement of the orientation of the magneticfield component of the ICME 10 hours prior to the ICME's arrival at Earth. This illustrates the space weather advance warning potential of FR imaging. In the case of the Halloween event, the 15 minute warning from in-situ magnetic-field measurements can be significantly improved to 10 hours advance warning.

\section{Results}

In summary, the boundary for investigating either the shock/sheath or the ICME driving it is where:

$$
\frac{\left\langle N_{\text {rope }} B_{p, \text { rope }}\right\rangle}{\left\langle N_{\text {sheath }} B_{p \text {, sheath }}\right\rangle}=1 .
$$

The basic configuration of the magnetic field in the ICME can be determined where this ratio is greater than one in the POS and used to determine the geoeffectiveness of the incoming ICME; however, the RM image simulations reveal that extensive investigation into the magnetic-field configuration of the shock/sheath structure would be possible with the MWA. For advance warning predictions on the effect of the shock/sheath, the best regions of the sheath to measure would be where the ratio in Equation (3) is significantly less than one; this would be outside of the flux rope structure itself. We also find that following the passage of the ICME with detailed in-situ magnetic-field and electron density measurements, MWA images could potentially support research into shock/sheath plasma flows as well as the evolution of the shock/sheath/ICME structure as it convects out into the heliosphere.

As noted previously, the MWA can obtain the measurements for a FR image at a single frequency between 80 to $300 \mathrm{MHz}$. When measuring FR, an ambiguity in the plane of polarization develops when the rotation exceeds \pm 180 degrees. The simulations shown in 
Figure 11 used an $R^{-2}$ heliocentric distance fall-off in density and magnetic-field strength; under these ideal conditions, we find that the \pm 180 degree ambiguity occurs at less than 15 solar radii for $300 \mathrm{MHz}$ and around 25 solar radii for $80 \mathrm{MHz}$. These values only apply to the CME and surrounding structure, not the static background heliospheric contribution. Note that the eight CMEs shown in Figure 1 did not show the existence of a shock/sheath region within $10 \mathrm{Rs}$; this suggests that a majority of CMEs do not develop a shock/sheath until reaching further heliospheric distances. For space weather prediction applications, we recommend the MWA measure FR at $300 \mathrm{MHz}$ to reduce the shock/sheath contribution to the signal.

\section{Summary}

The Committee on the Societal and Economic Impacts of Severe Space Weather Events, 2008, estimated a "severe geomagnetic storm scenario" triggered by a fast, geoeffective ICME could incur $\$ 1$ trillion in societal and economic damage. The NOAA Space Weather Center issues warnings regarding CME eruptions from the Sun; however, the geoeffectiveness of the ICME cannot be accurately quantified until the spacecraft in the $L_{1}$ libration point sunward of the Earth provide in-situ magnetic-field measurements. This gives an advance warning time of approximately an hour depending on ICME speed; in the case of the Halloween event, this was 15 minutes. The only remote sensing observing technique that can determine the magnetic-field orientation earlier and extend this advance warning time is Faraday rotation.

We demonstrated with spacecraft FR observations of CME/ICMEs that the critical parameters of the orientation and radius of a flux cylinder can be determined; however, the orientation measurement from a single LOS is degenerate. Using the capabilities of the MWA to investigate the potential uses for RM images, we find that the magnetic-field configuration in a CME/ICME can be uniquely determined. We also find that RM images pose an unparalleled opportunity to investigate the configuration of the magnetic field within the shock/sheath region on the leading edge of fast CME/ICMEs. These shock/sheath regions can potentially produce a RM response of similar strength as the flux cylinder; therefore, we recommend measuring FR at the greatest frequency possible thus observing the CME at the smallest heliospheric distance possible. Using the 29 October 2003 Halloween to study the use of RM images in increasing the advance warning time of accurate geoeffectiveness from magnetic-field measurements, we find an order of magnitude improvement from the average one hour to ten hours.

Acknowledgements The authors thank Christopher Russell, Joseph Salah, Justin Kasper, and Lan Jian. This research was supported by AFOSR/SFRL FA9550-06-1-0107, NSF ATM0925023, and NASA NNG05GM58G and NSF ATM0852240.

Open Access This article is distributed under the terms of the Creative Commons Attribution Noncommercial License which permits any noncommercial use, distribution, and reproduction in any medium, provided the original author(s) and source are credited.

\section{References}

Anselmo, J.C.: 1997, Solar storm eyed as satellite killer. Aviat. Week Space Technol. 146, 61.

Badruddin, Singh, Y.P.: 2009, Geoeffectiveness of magnetic cloud, shock/sheath, interaction region, high-speed stream and their combined occurrence. Planet. Space Sci. 57, 318-331. doi:10.1016/ j.pss.2008.12.009. 
Bird, M.K., Volland, H., Howard, R.A., Koomen, M.J., Michels, D.J., Sheeley, N.R. Jr., Amstrong, J.W., Seidel, B.L., Stelzried, C.T., Woo, R.: 1985, White-light and radio sounding observations of coronal transients. Solar Phys. 98, 341 - 368. doi:10.1007/BF00152465.

Cannon, A.R.: 1976, Radio frequency probing of the solar corona. PhD thesis, California Univ., Berkeley.

Cliver, E.W., Svalgaard, L.: 2004, The 1859 Solar-terrestrial disturbance and the current limits of extreme space weather activity. Solar Phys. 224, 407-422. doi:10.1007/s11207-005-4980-z.

Committee on the Societal, Economic Impacts of Severe Space Weather Events: 2008, Severe space weather events - Understanding societal and economic impacts. A Workshop Report. Technical report.

Dunn, T., Jackson, B.V., Hick, P.P., Buffington, A., Zhao, X.P.: 2005, Comparative analyses of the CSSS calculation in the UCSD tomographic solar observations. Solar Phys. 227, 339-353. doi:10.1007/s11207-005-2759-x.

Forsyth, R.J., Bothmer, V., Cid, C., Crooker, N.U., Horbury, T.S., Kecskemety, K., Klecker, B., Linker, J.A., Odstrcil, D., Reiner, M.J., Richardson, I.G., Rodriguez-Pacheco, J., Schmidt, J.M., WimmerSchweingruber, R.F.: 2006, ICMEs in the inner heliosphere: Origin, evolution and propagation effects. Report of working group G. Space Sci. Rev. 123, 383 -416. doi:10.1007/s11214-006-9022-0.

Glaser, D., Beals, K., Pompea, S., Willard, C.: 2003, Living with a Star: GEMS Teacher's Guide Website, The Regents of the University of California. http://ds9.ssl.berkeley.edu/lws_gems/index.htm.

Jackson, B.V., Buffington, A., Hick, P.P., Wang, X., Webb, D.: 2006, Preliminary three-dimensional analysis of the heliospheric response to the 28 October 2003 CME using SMEI white-light observations. J. Geophys. Res. (Space Phys.) 111, 4. doi:10.1029/2004JA010942.

Jensen, E.A., Russell, C.T.: 2008, Faraday rotation observations of CMEs. Geophys. Res. Lett. 35, 2103. doi:10.1029/2007GL031038.

Kane, R.P.: 2007, Solar terrestrial effects of two distinct types. Adv. Space Res. 39, 1890 - 1897. doi:10.1016/ j.asr.2007.02.006.

Levy, G.S., Sato, T., Seidel, B.L., Stelzried, C.T., Ohlson, J.E., Rusch, W.V.T.: 1969, Pioneer 6: Measurement of transient Faraday rotation phenomena observed during solar occultation. Science 166, 596-598. doi:10.1126/science.166.3905.596.

Lindsay, G.M., Luhmann, J.G., Russell, C.T., Gosling, J.T.: 1999, Relationships between coronal mass ejection speeds from coronagraph images and interplanetary characteristics of associated interplanetary coronal mass ejections. J. Geophys. Res. 104, 12515 - 12524. doi:10.1029/1999JA900051.

Liu, Y., Manchester, W.B., Richardson, J.D., Luhmann, J.G., Lin, R.P., Bale, S.D.: 2008, Deflection flows ahead of ICMEs as an indicator of curvature and geoeffectiveness. J. Geophys. Res. (Space Phys.) 113, A00B03. doi:10.1029/2007JA012996.

Livesey, R.J.: 1998, The Sun, interplanetary weather and mankind's technologies. J. Br. Astron. Assoc. 108, $207-209$.

Mulligan, T., Russell, C.T.: 2001, Multispacecraft modeling of the flux rope structure of interplanetary coronal mass ejections: Cylindrically symmetric versus nonsymmetric topologies. J. Geophys. Res. 106, 10581 10596. doi:10.1029/2000JA900170.

Mulligan, T.L.: 2002, The structure of interplanetary coronal mass ejections and their solar origins. PhD thesis, University of California, Los Angeles.

Nelder, J.A., Mead, R.: 1965, A simplex method for function minimization. Comput. J. 7, $308-313$. doi:10.1093/comjnl/7.4.308.

NOAA Space Weather Prediction Center: 2003, ACE real time solar wind news and announcements. Technical report. http://www.swpc.noaa.gov/ace.

Nordwall, B.D.: 1996, Atmospheric/multipath concerns for D-GPS. Aviat. Week Space Technol. 145, 60.

Odenwald, S.: 1999, Solar storms. The Washington Post, H01.

Russell, C.T., Mulligan, T.: 2003, The limitation of Bessel functions for ICME modeling. In: Velli, M., Bruno, R., Malara, F., Bucci, B. (eds.) Solar Wind Ten, AIP Conf. Proc. 679, 125 - 128.

Salah, J.E., Lonsdale, C.J., Oberoi, D., Cappallo, R.J., Kasper, J.C.: 2005, Space weather capabilities of low frequency radio arrays. In: Fineschi, S., Viereck, R.A. (eds.) SPIE Conf. Ser. 5901, 124-134. doi:10.1117/12.613448.

Skoug, R.M., Gosling, J.T., Steinberg, J.T., McComas, D.J., Smith, C.W., Ness, N.F., Hu, Q., Burlaga, L.F.: 2004, Extremely high speed solar wind: 29-30 October 2003. J. Geophys. Res. (Space Phys.) 109, 9102. doi:10.1029/2004JA010494.

Smith, E.A.: 1988, Correlations between solar activity and operationally determined satellite drag variation parameters. In: Flight Mechanics/Estimation Theory Symposium, 571 - 594.

Stelzried, C.T.: 1968, A Faraday rotation measurement of a 13-cm signal in the solar corona. Technical Report 32-1401, Jet Propulsion Laboratory, Pasadena, CA 91109.

Subramanian, P., Vourlidas, A.: 2007, Energetics of solar coronal mass ejections. Astron. Astrophys. 467, 685-693. doi:10.1051/0004-6361:20066770. 
Taylor, J.B.: 1986, Relaxation and magnetic reconnection in plasmas. Rev. Mod. Phys. 58, 741-763. doi:10.1103/RevModPhys.58.741.

Tokumaru, M., Kojima, M., Fujiki, K., Yamashita, M., Jackson, B.V.: 2007, The source and propagation of the interplanetary disturbance associated with the full-halo coronal mass ejection on 28 October 2003. J. Geophys. Res. (Space Phys.) 112, 5106. doi:10.1029/2006JA012043. 